\title{
Test of the generalized Brink-Axel hypothesis in ${ }^{64,65} \mathrm{Ni}$
}

\author{
L. Crespo Campo, ${ }^{1, *}$ M. Guttormsen, ${ }^{1}$ F. L. Bello Garrote, ${ }^{1}$ T. K. Eriksen, ${ }^{2}$ F. Giacoppo, ${ }^{3,4}$ A. Görgen, ${ }^{1}$ K. Hadynska-Klek, ${ }^{5}$ \\ M. Klintefjord, ${ }^{1}$ A. C. Larsen, ${ }^{1}$ T. Renstrøm, ${ }^{1}$ E. Sahin, ${ }^{1}$ S. Siem, ${ }^{1}$ A. Springer, ${ }^{6}$ T. G. Tornyi, ${ }^{2}$ and G. M. Tveten ${ }^{1}$ \\ ${ }^{1}$ Department of Physics, University of Oslo, N-0316 Oslo, Norway \\ ${ }^{2}$ Department of Nuclear Physics, Research School of Physics and Engineering, The Australian National University, Acton, \\ Australia Capital Territory 2601, Australia \\ ${ }^{3}$ Helmholtz Institute Mainz, 55099 Mainz, Germany \\ ${ }^{4}$ GSI Helmholtzzentrum für Schwerionenforschung GmbH, 64291 Darmstadt, Germany \\ ${ }^{5}$ Istituto Nazionale di Fisica Nucleare, Laboratori Nazionali di Legnaro, 235020 Legnaro (Padua), Italy \\ ${ }^{6}$ Department of Physics, Karlsruhe Institute of Technology, D-76131 Karlsruhe, Germany
}

(Received 23 June 2017; revised manuscript received 18 February 2018; published 6 November 2018)

\begin{abstract}
Previously published particle- $\gamma$ coincidence data on the ${ }^{64} \mathrm{Ni}\left(p, p^{\prime} \gamma\right){ }^{64} \mathrm{Ni}$ and ${ }^{64} \mathrm{Ni}(d, p \gamma){ }^{65} \mathrm{Ni}$ reactions were further analyzed to study the statistical properties of $\gamma$ decay in ${ }^{64,65} \mathrm{Ni}$. To do so, the $\gamma$ decay to the quasicontinuum region and discrete low-lying states was investigated at $\gamma$-ray energies of 2.0-9.6 and 1.6-6.1 MeV in ${ }^{64} \mathrm{Ni}$ and ${ }^{65} \mathrm{Ni}$, respectively. In particular, the dependence of the $\gamma$-strength function with initial and final excitation energy was studied to test the validity of the generalized Brink-Axel hypothesis. Finally, the role of fluctuations in transition strengths was estimated as a function of $\gamma$-ray and excitation energy. The $\gamma$-strength function is consistent with the hypothesis of the independence of initial excitation energy, in accordance with the generalized Brink-Axel hypothesis. The results show that the $\gamma$ decay to low-lying levels displays large fluctuations for low initial excitation energies.
\end{abstract}

DOI: 10.1103/PhysRevC.98.054303

\section{INTRODUCTION}

Many analytical techniques in nuclear physics are based on the hypothesis of nuclear $\gamma$ decay from a compound nuclear state: it is assumed that a given reaction has led to a compound state which decays independently of how it was formed [1]. At sufficiently high excitation energies, the nucleus is considered to be in its quasicontinuum regime, a region where the number of nuclear states is so high that they mix strongly with each other. At that point, nuclear $\gamma$ decay is studied as an average of a large number of transitions and thus two statistical properties can be defined: the nuclear level density (NLD), or number of nuclear states per unit of energy, and the $\gamma$-strength function $(\gamma \mathrm{SF})$, or average reduced $\gamma$-ray transition probability [2]. Furthermore, the calculations are often simplified by assuming that the generalized Brink-Axel (gBA) hypothesis is valid. In general terms, the gBA hypothesis implies that the dipole $\gamma$ strength is independent of the structure of the initial state; i.e, it has no explicit dependence on the excitation energy, spin, or parity, except for the obvious selection rules for dipole transitions $[3,4]$.

When the gBA hypothesis holds, the $\gamma$ SF depends solely on the $\gamma$-ray energy for dipole radiation [2]. The gBA hypothesis simplifies the description of nuclear $\gamma$ and $\beta$ decays, being frequently used in the calculation of neutron-capture cross sections [5-8]. Determining the circumstances under which the gBA hypothesis holds is therefore of great importance, due

\footnotetext{
*1.c.campo@fys.uio.no
}

to its impact in fields such as nuclear astrophysics and reactor physics.

The experimental and theoretical attempts made to validate the gBA hypothesis demonstrate that it is a question of great complexity. Some of the first verifications of this hypothesis below nucleon-emission thresholds come from the analysis of mainly $E 1$ high-energy primary transitions, either from isolated resonances or from average neutron-capture data [9]. In addition, the observations of $M 1$ scissors modes in ${ }^{163} \mathrm{Dy}$ [10] and ${ }^{172} \mathrm{Yb}$ [11], deduced from radiative neutron-capture measurements, also support this hypothesis. Furthermore, the results from Refs. [12,13] show that, for a heavy odd-odd nucleus such as ${ }^{238} \mathrm{~Np}$, the NLD is extremely high and averaging over many $\gamma$ transitions can be performed. Under these circumstances, there is a significant suppression of PorterThomas [14] fluctuations, i.e, fluctuations in the individual partial radiative widths related to the complexity of the nuclear states involved in the decay. Thus, the gBA hypothesis could be reliably tested in ${ }^{238} \mathrm{~Np}$. For lighter nuclei, however, the situation is normally rather different and large Porter-Thomas fluctuations are present $[13,15]$. Such fluctuations can be understood in terms of the wave functions of the various nuclear states involved in a given decay. The reduced width amplitude depends on the wave functions of both the compound nuclear state and the exit channel. If these wave functions are of great complexity, the statistical model can be applied: the strengths of $\gamma$-ray transitions (or rather the partial radiative widths) are assumed to follow a Porter-Thomas distribution and the various exit channels are assumed to be independent [14]. For sufficiently heavy nuclei and/or when high excitation energies 
are involved, the complexity of the nuclear wave functions allows for the application of such a statistical model hypothesis. However, for light nuclei the lower NLD (and lower complexity of the involved wave functions) can challenge these statistical descriptions and result in large fluctuations of the radiative widths [16], thus making the validation of the gBA hypothesis difficult.

Despite the continued interest in the gBA hypothesis, the available information regarding this matter is still quite scarce. More experimental data on $\gamma$-decay properties would help in understanding the connection between the validity of this hypothesis and nuclear structure phenomena. Furthermore, these measurements could greatly improve the estimation of $(n, \gamma)$ cross sections, which are often highly uncertain for unstable neutron-rich nuclei $[17,18]$.

In this work, previously published particle- $\gamma$ coincidence data on the ${ }^{64} \mathrm{Ni}\left(p, p^{\prime} \gamma\right){ }^{64} \mathrm{Ni}$ and ${ }^{64} \mathrm{Ni}(d, p \gamma){ }^{65} \mathrm{Ni}$ reactions from Refs. $[19,20]$ were further analyzed to study the dependence of the $\gamma \mathrm{SF}$ with initial and final excitation energy. In other words, we investigated the energy regions under which the use of an excitation-energy-independent $\gamma \mathrm{SF}$ is appropriate and thus tested the validity of the gBA hypothesis.

This article is organized as follows: In Sec. II the experimental details and analytical method are summarized and the new results presented. The $\gamma \mathrm{SF}$ for different excitation energies is studied in Secs. II A and IIB. In Sec. III the fluctuations of the $\gamma \mathrm{SF}$ are discussed. Finally, a summary is given in Sec. IV.

\section{THE $\gamma$ SF OF ${ }^{64,65} \mathrm{Ni}$ AND ITS DEPENDENCE ON EXCITATION ENERGY}

\section{A. The standard Oslo method applied to various energy regions}

The data employed in this work were first presented in Refs. [19,20], where details on the experimental method are given. As shown there, particle- $\gamma$ coincidences were measured and used to obtain coincidence matrices for ${ }^{64} \mathrm{Ni}$ and ${ }^{65} \mathrm{Ni}$, in which the $\gamma$ rays emitted from a given excitation energy are represented. Applying the Oslo method [21-24], the spectra were unfolded [21] and the first-generation (primary) $\gamma$ rays were extracted [22].

A section in the primary matrix was selected to extract data corresponding to statistical $\gamma$ decay from compound nuclear states, for which the gBA hypothesis was expected to hold $[3,25]$. Then, the selected region in the primary matrix was used to obtain the functional form of the NLD $\rho$ [26] and the $\gamma$-transmission coefficient [2] $\mathscr{T}$ through

$$
P\left(E_{\gamma}, E_{i}\right) \propto \rho\left(E_{f}\right) \mathscr{T}\left(E_{\gamma}\right),
$$

where $P\left(E_{\gamma}, E_{i}\right)$ represents the probability of $\gamma$ decay from an initial excitation energy $E_{i}$ with a $\gamma$-ray energy $E_{\gamma}$, obtained from the primary coincidence matrix $U$ as $P\left(E_{\gamma}, E_{i}\right)=U\left(E_{\gamma}, E_{i}\right) / \sum_{E_{\gamma},} U\left(E_{\gamma}, E_{i}\right) . E_{f}$ is the final excitation energy, with $E_{f}=E_{i}-E_{\gamma}$. From $\mathscr{T}$, the $\gamma \mathrm{SF}$ was obtained with the relation $[27,28]$

$$
f_{L}\left(E_{\gamma}\right)=\frac{\mathscr{T}\left(E_{\gamma}\right)}{2 \pi E_{\gamma}^{2 L+1}},
$$

where $L$ is the multipolarity of the transition, here taken as $L=1$ since dipole radiation is expected to represent the main contribution to the $\gamma \mathrm{SF}$ in the quasicontinuum [27,29]. The NLD and $\gamma \mathrm{SF}$ were then normalized using additional experimental data.

The primary coincidence matrices for ${ }^{64,65} \mathrm{Ni}$ obtained in Refs. $[19,20]$ are included in Figs. 1(a) and 2(a). The regions used for the extraction of the NLD and $\gamma \mathrm{SF}$ are also shown for the standard Oslo method. For ${ }^{64} \mathrm{Ni}$, the region corresponds to $E_{\gamma}>1.98 \mathrm{MeV}$ and $E_{x}=5.82-9.66 \mathrm{MeV}$ [19], while for ${ }^{65} \mathrm{Ni}$ the chosen limits were $E_{\gamma}>1.60 \mathrm{MeV}$ and $E_{x}=4.43-6.08 \mathrm{MeV}$ [20]. The resulting $\gamma \mathrm{SFs}$ are shown in Figs. 1(b) and 2(b). The excitation energy resolution (FWHM) is $\approx 130 \mathrm{keV}$ at $692 \mathrm{keV}$ and it is approximately constant with excitation energy, while the $\gamma$-ray energy resolution is $\approx 70 \mathrm{keV}$ at $1017 \mathrm{keV}$ and scales with $\sqrt{E_{\gamma}}$.

As seen in Figs. 1 and 2, the selected region contains a large area for which the data vary smoothly with excitation and $\gamma$ energy and thus the decay from compound nuclear states is expected to dominate. Nevertheless, strong diagonals
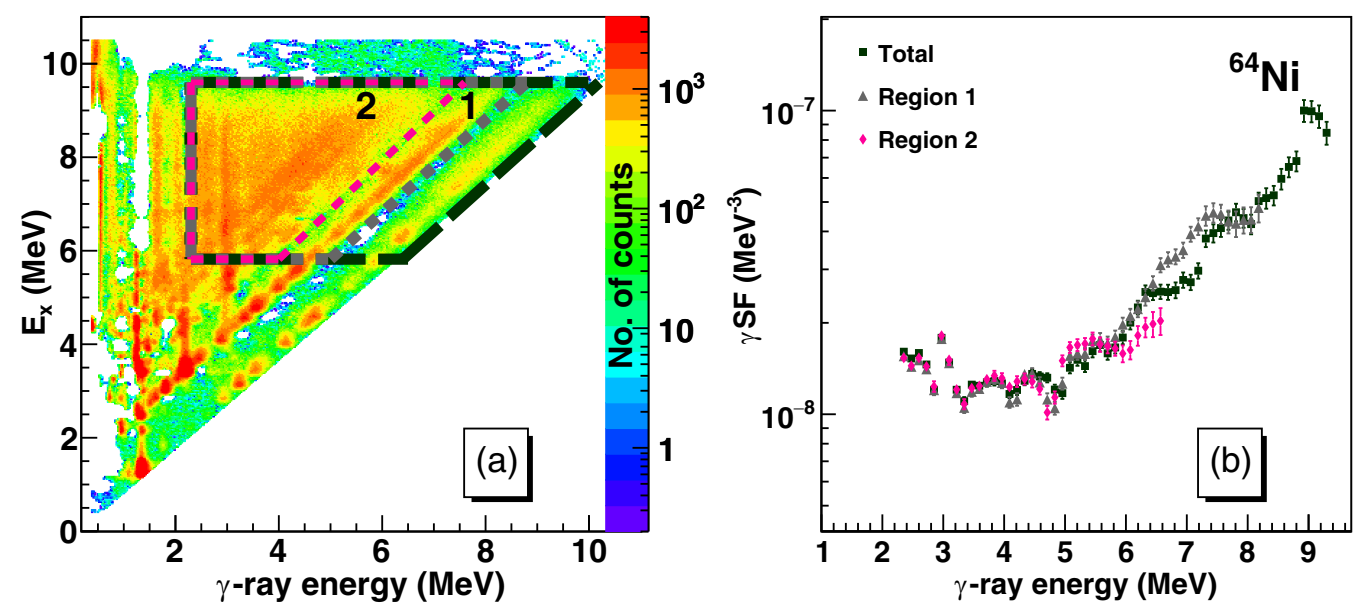

FIG. 1. The $\gamma \mathrm{SF}$ as obtained from different regions in the first-generation coincidence matrix for ${ }^{64} \mathrm{Ni}$ : (a) the considered regions in the coincidence matrix and (b) the resulting $\gamma$ SF. The total region corresponds to the standard $\gamma$ SF as obtained in Ref. [19]. 

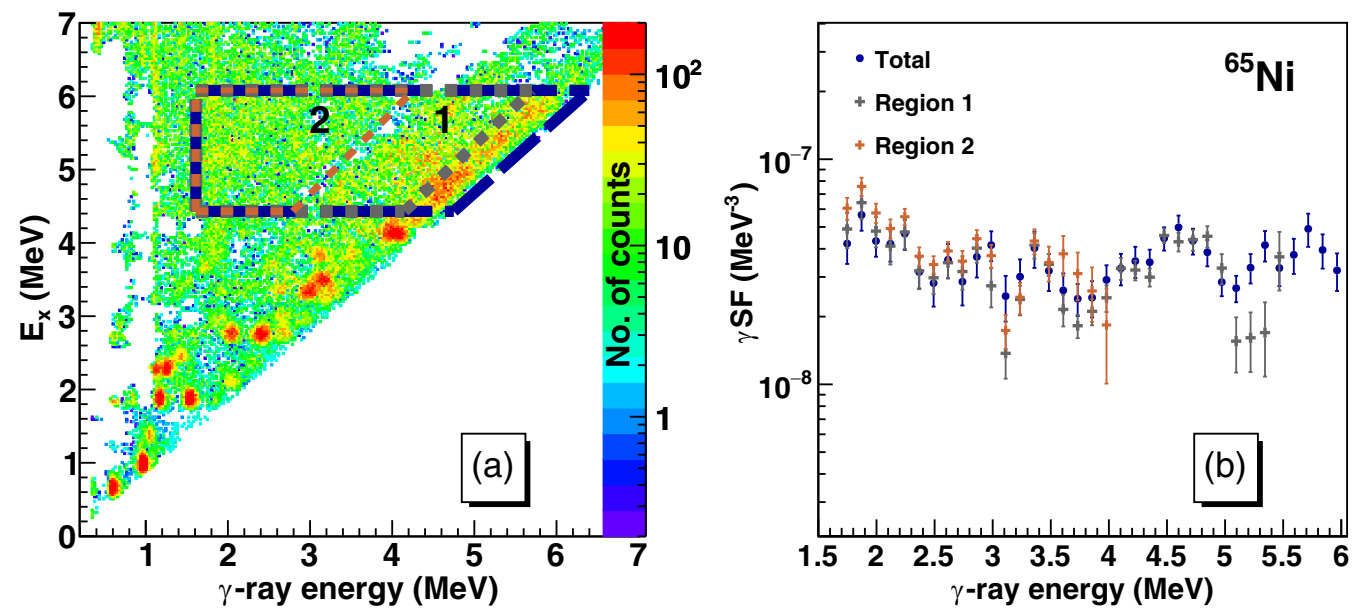

FIG. 2. The $\gamma \mathrm{SF}$ obtained from different regions in the first-generation coincidence matrix for ${ }^{65} \mathrm{Ni}$ [20]: (a) the considered regions in the coincidence matrix and (b) the resulting $\gamma \mathrm{SF}$. The total region corresponds to the standard $\gamma \mathrm{SF}$.

are present. In the case of ${ }^{64} \mathrm{Ni}$, a diagonal at $E_{x} \approx E_{\gamma}$ is seen, corresponding to the decay to the ground state. A much stronger feeding to the first excited $2^{+}$state is measured, corresponding to the diagonal at $E_{x} \approx E_{\gamma}-1.345 \mathrm{MeV}$ [19]. For ${ }^{65} \mathrm{Ni}$, the most pronounced diagonal is observed at $E_{x} \approx$ $E_{\gamma}$, indicating the presence of strong $\gamma$-ray transitions feeding both the ground state $5 / 2^{-}$and the first excited $1 / 2^{-}$isomeric state at $63.37 \mathrm{keV}$. Since the neutron separation energy $S_{n}$ for ${ }^{65} \mathrm{Ni}$ is considerably lower $\left(S_{n}=6.098 \mathrm{MeV}\right)$ than for ${ }^{64} \mathrm{Ni}$, these $\gamma$-decay transitions are strong even at excitation energies very close to $S_{n}$ [20].

To study the impact of these transitions in the $\gamma \mathrm{SFs}$ of ${ }^{64,65} \mathrm{Ni}$, the Oslo method was applied to smaller and smoother regions of the first-generation coincidence matrix. This technique was used for ${ }^{65} \mathrm{Ni}$ in Ref. [20] as shown in Fig. 2. In this work, the same technique was applied to ${ }^{64} \mathrm{Ni}$ and the results are included in Fig. 1. The regions used for the analysis are depicted in Figs. 1(a) and 2(a), while the resulting $\gamma$ SFs are shown in Figs. 1(b) and 2(b). The results are compared to the standard $\gamma$ SF obtained in Refs. $[19,20]$.

For both ${ }^{64} \mathrm{Ni}$ and ${ }^{65} \mathrm{Ni}$, the strong diagonal at $E_{x} \approx E_{\gamma}$ was excluded in region 1 . Furthermore, a smaller section of the matrix (region 2) was selected and the corresponding $\gamma \mathrm{SF}$ included. In the case of ${ }^{64} \mathrm{Ni}$, region 2 is obtained when excluding the strong diagonal that corresponds to the feeding of the first excited $2^{+}$state at $1.345 \mathrm{MeV}$.

As seen in Fig. 1 for ${ }^{64} \mathrm{Ni}$, the $\gamma \mathrm{SF}$ obtained from regions 1 and 2 are in good agreement with the standard $\gamma \mathrm{SF}$ at $\gamma$-ray energies below $\approx 6.0 \mathrm{MeV}$, although some deviations are seen at $E_{\gamma} \approx 5-6.0 \mathrm{MeV}$. At $E_{\gamma} \approx 6.0-7.6 \mathrm{MeV}$, the results from region 1 are substantially higher than the standard ones. Based solely on the results from Fig. 1, this could be interpreted as being partially due to the strong feeding of the first excited state: in the standard $\gamma \mathrm{SF}$, the results with $E_{\gamma}>6.0 \mathrm{MeV}$ include $\gamma$ decays to both the ground and the first excited $2^{+}$states, while in the results from region 1 the decay to the ground state is excluded. As seen from the coincidence matrix, the feeding of the first excited state is stronger than for the ground state, and thus this could be seen as being due to a standard $\gamma \mathrm{SF}$ which is lower than for region 1 above $E_{\gamma} \approx 6.0 \mathrm{MeV}$. However, the coincidence matrix contains contributions from both the NLD and the $\gamma \mathrm{SF}$ and, as shown later in Fig. 7, the $\gamma \mathrm{SF}$ feeding the first excited state is perfectly compatible with the standard $\gamma \mathrm{SF}$, at least for $E_{i}>6 \mathrm{MeV}$. This leads us to the conclusion that the discrepancies observed in Fig. 1(b) between the $\gamma \mathrm{SFs}$ at $E_{\gamma}>$ $6 \mathrm{MeV}$ are most likely due to differences in the normalizations for the various regions, which cannot be exactly the same with this particular procedure. Furthermore, differences in the normalization are more likely to impact the resulting $\gamma \mathrm{SFs}$ at $\gamma$-ray energies approaching $E_{\gamma} \approx S_{n}-E_{\gamma \text {,low }}$ and at $E_{\gamma}>$ $S_{n}-E_{\gamma, \text { low }}$. This is due to the applied extrapolation at such energies, where $E_{\gamma, \text { low }}$ is the minimum value of $E_{\gamma}$ used in the extraction of the $\gamma$ SF via the Oslo method $[19,24]$. The results presented in Fig. 1 suggest that the $\gamma \mathrm{SF}$ for ${ }^{64} \mathrm{Ni}$ obtained in Ref. [19] (the standard $\gamma \mathrm{SF}$ ) is indeed a good estimate for $E_{\gamma}<6.0 \mathrm{MeV}$. Above that energy, the standard $\gamma \mathrm{SF}$ is most likely to be the best estimate as well, extracted using all the available data (total region in Fig. 1).

As shown in Fig. 2 the results for ${ }^{65} \mathrm{Ni}$ are in very good agreement with the standard $\gamma \mathrm{SF}$ [20]. The low-energy enhancement or upbend ${ }^{1}$ is reproduced and the analysis confirms that the resonancelike structure at $E_{\gamma}=4.6 \mathrm{MeV}$ is truly present and that it is not just the result of a strong feeding to the ground state.

\footnotetext{
${ }^{1}$ The expressions low-energy enhancement and upbend are here used to describe an increase in the $\gamma \mathrm{SF}$ with decreasing $\gamma$-ray energy at energies below $E_{\gamma} \approx 2-4 \mathrm{MeV}$. An enhancement is observed below $E_{\gamma} \approx 3 \mathrm{MeV}$ for ${ }^{64,65} \mathrm{Ni}[19,20]$ when compared to the descriptions given by Lorentzian models such as the standard Lorentzian [27], which predicts a decrease in the $\gamma \mathrm{SF}$ at low $\gamma$ ray energies. The low-energy enhancement is also observed when compared to the predictions from the generalized Lorentzian for ${ }^{64} \mathrm{Ni}$, as shown in Ref. [19].
} 


\section{B. The $\gamma \mathrm{SF}$ as a function of initial and final states}

To test the validity of the gBA hypothesis, the dependence of the $\gamma$ SF with both initial and final excitation energy was studied applying the technique described in Ref. [12]. Assuming that the $\gamma$-transmission coefficient $\mathscr{T}$ only depends on $E_{\gamma}$, we introduce a normalization factor $N$ which only depends on the initial excitation energy and rewrite Eq. (1) as

$$
N\left(E_{i}\right) P\left(E_{\gamma}, E_{i}\right)=\rho\left(E_{i}-E_{\gamma}\right) \mathscr{T}\left(E_{\gamma}\right),
$$

which determines the normalization factor by

$$
N\left(E_{i}\right)=\frac{\int_{0}^{E_{i}} \mathscr{T}\left(E_{\gamma}\right) \rho\left(E_{i}-E_{\gamma}\right) d E_{\gamma}}{\int_{0}^{E_{i}} P\left(E_{\gamma}, E_{i}\right) d E_{\gamma}} .
$$

Since $\rho$ is known, $\mathscr{T}$ can be studied in detail for each excitation energy bin by $N P / \rho$, as given in Eq. (3). Therefore, for the initial excitation energy states we obtain [12]

$$
\mathscr{T}\left(E_{\gamma}, E_{i}\right)=\frac{N\left(E_{i}\right) P\left(E_{\gamma}, E_{i}\right)}{\rho\left(E_{i}-E_{\gamma}\right)} .
$$

In a similar way, we define $\mathscr{T}\left(E_{\gamma}, E_{f}\right)$ and study the decay to final excitation energies:

$$
\mathscr{T}\left(E_{\gamma}, E_{f}\right)=\frac{N\left(E_{f}+E_{\gamma}\right) P\left(E_{\gamma}, E_{f}+E_{\gamma}\right)}{\rho\left(E_{f}\right)} .
$$

The normalization factor $N$ is obtained assuming that both $\mathscr{T}\left(E_{\gamma}, E_{i}\right)$ and $\mathscr{T}\left(E_{\gamma}, E_{f}\right)$ fluctuate around the excitationenergy-independent $\mathscr{T}\left(E_{\gamma}\right)$ obtained with the standard Oslo method from Eqs. (1) and (4). Applying the relation between the $\gamma \mathrm{SF}$ and $\mathscr{T}$ given by Eq. (2), the corresponding $\gamma \mathrm{SF}$ functions for the initial and final states, $f\left(E_{\gamma}, E_{i}\right)$ and $f\left(E_{\gamma}, E_{f}\right)$, were obtained and averaged over the initial and final excitation energies by [12]

$$
\begin{aligned}
f_{i}\left(E_{\gamma}\right) & =\frac{1}{S_{n}-E_{\gamma}} \int_{E_{\gamma}}^{S_{n}} f\left(E_{\gamma}, E_{i}\right) d E_{i}, \\
f_{f}\left(E_{\gamma}\right) & =\frac{1}{S_{n}-E_{\gamma}} \int_{0}^{S_{n}-E_{\gamma}} f\left(E_{\gamma}, E_{f}\right) d E_{f},
\end{aligned}
$$

where $E_{\gamma}>1.98 \mathrm{MeV}$ and $S_{n}=9.66 \mathrm{MeV}$ for ${ }^{64} \mathrm{Ni}$ while $E_{\gamma}>1.60 \mathrm{MeV}$ and $S_{n}=6.08 \mathrm{MeV}$ for ${ }^{65} \mathrm{Ni}[19,20,30]$.

To check that the normalization function $N\left(E_{i}\right)$ is reasonable, the results for $f_{i}\left(E_{\gamma}\right), f_{f}\left(E_{\gamma}\right)$, and the $\gamma$ SFs obtained with the standard Oslo method $f\left(E_{\gamma}\right)$ were compared, as shown in Figs. 3 and 4. The functions are in good agreement, supporting the applied normalization function.

With the well-behaving normalization function, the $\gamma \mathrm{SF}$ was studied as a function of initial and final excitation energies. The dependence of the $\gamma \mathrm{SF}$ with initial excitation energy $f\left(E_{\gamma}, E_{i}\right)$ is shown for ${ }^{64,65} \mathrm{Ni}$ in Figs. 5 and 6, respectively. Note that each $f\left(E_{\gamma}, E_{i}\right)$ is built on a given initial excitationenergy gate, but with no specific final state. However, for a given $E_{\gamma}$ and $E_{i}$, the final excitation energy is determined as $E_{f}=E_{i}-E_{\gamma}$. The $\gamma$ SFs obtained with the standard Oslo method, $f\left(E_{\gamma}\right)$, are also shown in Figs. 5 and 6 (solid line). The agreement between the obtained $f\left(E_{\gamma}, E_{i}\right)$ and the standard strength is clear for both ${ }^{64,65} \mathrm{Ni}$. The uncertainties are, however, large for ${ }^{65} \mathrm{Ni}$, due to lower statistics. The results

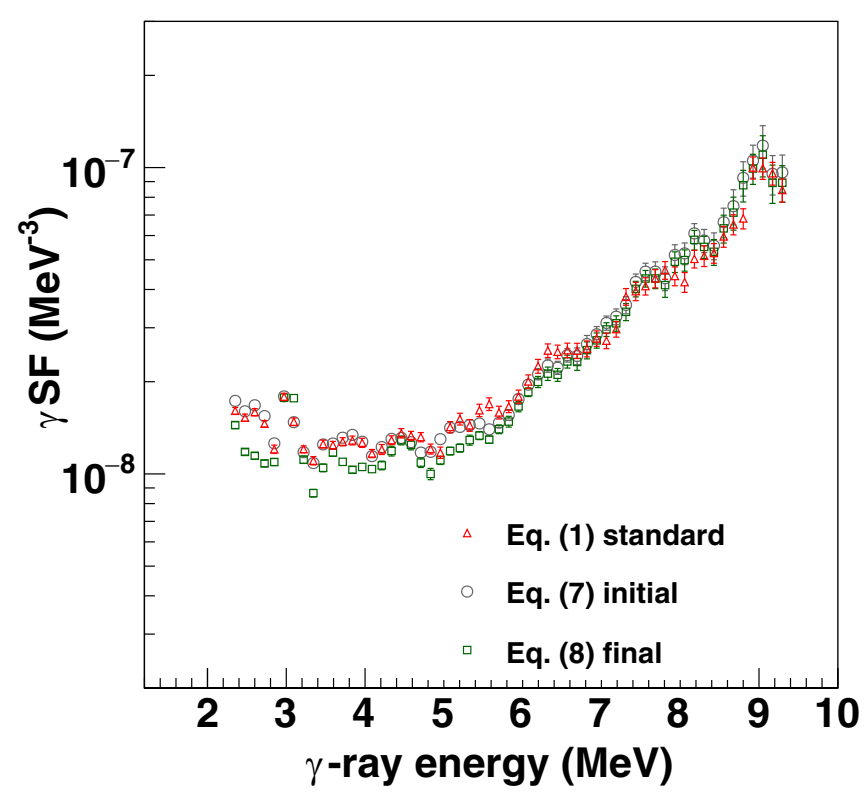

FIG. 3. Comparison of $f\left(E_{\gamma}\right), f_{i}\left(E_{\gamma}\right)$, and $f_{f}\left(E_{\gamma}\right)$ for ${ }^{64} \mathrm{Ni}$.

for $f\left(E_{\gamma}, E_{i}\right)$ reproduce the structures present in the standard $\gamma \mathrm{SF}$ for both nuclei within uncertainties. Not only is the measured low-energy enhancement present for the various $E_{i}$, but its shape seems independent of $E_{i}$ for both ${ }^{64,65} \mathrm{Ni}$.

The resemblance between the individual $f\left(E_{\gamma}, E_{i}\right)$ with the standard $f\left(E_{\gamma}\right)$ is due to the averaging over a relatively high number of transitions, thus preventing large fluctuations. However, exploiting the decay to a few final states will enhance such fluctuations dramatically. Figure 7 shows the average $\gamma \mathrm{SF}$ feeding a given final excitation energy bin for ${ }^{64} \mathrm{Ni}, f\left(E_{\gamma}, E_{f}\right)$. The corresponding results for ${ }^{65} \mathrm{Ni}$ are shown in Fig. 8. In both cases the $E_{f}$ bins were chosen to

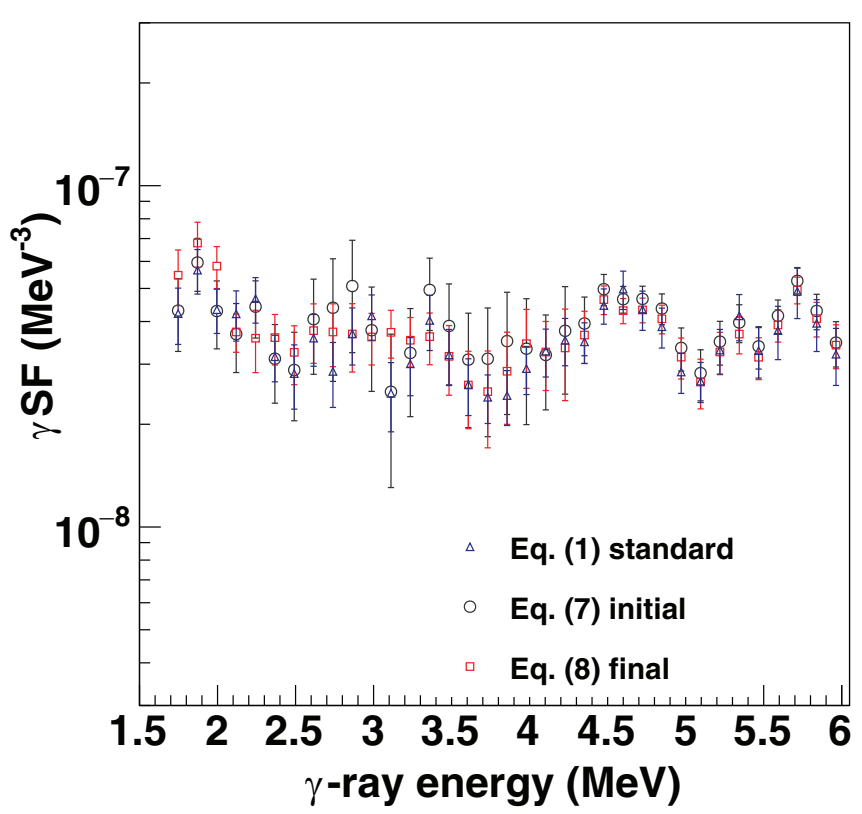

FIG. 4. Comparison of $f\left(E_{\gamma}\right), f_{i}\left(E_{\gamma}\right)$, and $f_{f}\left(E_{\gamma}\right)$ for ${ }^{65} \mathrm{Ni}$. 


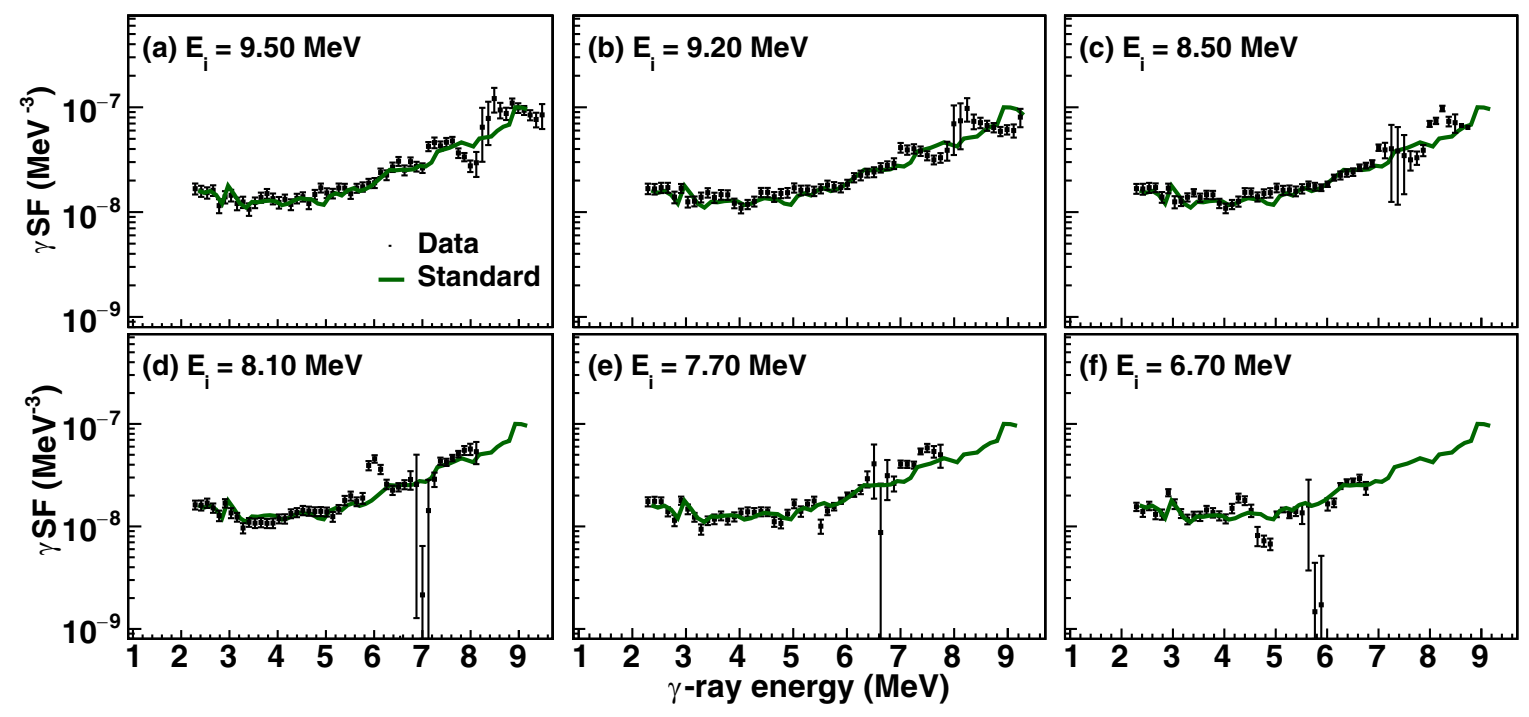

FIG. 5. $\gamma$ strength from several initial excitation energies in ${ }^{64} \mathrm{Ni}$. The results are compared to the standard $\gamma \mathrm{SF}$ (solid line). Each initial excitation energy bin has a width of $124 \mathrm{keV}$.

contain discrete energy levels, with widths of 124 and $248 \mathrm{keV}$ for ${ }^{64} \mathrm{Ni}$ and ${ }^{65} \mathrm{Ni}$, respectively. It is important to note that the standard $\gamma \mathrm{SF}, f\left(E_{\gamma}\right)$, is obtained at excitation energies above $E_{x, \min }=5.82$ and $4.43 \mathrm{MeV}$ for ${ }^{64} \mathrm{Ni}$ and ${ }^{65} \mathrm{Ni}$. At those excitation energies, the $\gamma \mathrm{SF}$ was expected to be independent of excitation energy $[19,20]$. Therefore, for a given $E_{f}$, the results for $f\left(E_{\gamma}, E_{f}\right)$ with $E_{\gamma}+E_{f}>E_{x \text {, min }} \mathrm{MeV}$ should be in agreement with the standard $\gamma \mathrm{SF}, f\left(E_{\gamma}\right)$. As observed in Figs. 7 and 8, this is the case for both ${ }^{64} \mathrm{Ni}$ and ${ }^{65} \mathrm{Ni}$, considering the presence of statistical uncertainties and fluctuations. Only two data points in Figs. 7(a) and 7(b) show larger deviations with respect to $f\left(E_{\gamma}\right)$, corresponding to decays from $E_{i} \approx 6.2-6.4 \mathrm{MeV}$. A vertical dashed line is included in Figs. 7 and 8 to indicate the limit $E_{\gamma}=E_{x \text {, min }}-E_{f}$.

In the case of ${ }^{64} \mathrm{Ni}$, the strengths feeding states above $E_{f} \approx$ $3.3 \mathrm{MeV}$ are in very good agreement with the standard $\gamma \mathrm{SF}$, as seen in Figs. 7(e) and 7(f). A good agreement is also seen for $E_{f}=2.972 \mathrm{MeV}$ at $E_{\gamma}>2.8 \mathrm{MeV}$. The feeding of the $2^{+}$state at $2.276 \mathrm{MeV}$ [30] also shows a similar trend as the standard $\gamma \mathrm{SF}$ above $E_{\gamma} \approx 3.5 \mathrm{MeV}$, although significant deviations are observed. In other words, the results for $f\left(E_{\gamma}, E_{f}\right)$ in Figs. 7(c) and 7(d) are in agreement with $f\left(E_{\gamma}\right)$ for $\gamma$-ray energies that correspond to $E_{i}>5.6-5.8 \mathrm{MeV}$, close to the lower limit of the $E_{i}$ range used for the extraction of $f\left(E_{\gamma}\right)$. This confirms that an excitation-energy-independent $\gamma \mathrm{SF}$ is a good assumption above $E_{i} \approx 5.8 \mathrm{MeV}$. As seen in Fig. 7(c), below $E_{\gamma} \approx 3.5 \mathrm{MeV}$ some fluctuations are present, although they are scattered around the standard $\gamma \mathrm{SF}$. Therefore, overall the results from Fig. 7 suggest that the $\gamma \mathrm{SF}$ is independent of $E_{f}$ at and above $\approx 3.0 \mathrm{MeV}$. The strength feeding the ground and first excited states presents very large fluctuations, which are scattered around the standard $\gamma \mathrm{SF}$. The presence of fluctuations is to be expected given the strong Porter-Thomas fluctuations. For instance, at $E_{i}=3.3-4 \mathrm{MeV}$, only three transitions to the ground state have been seen according to Ref. [30]. As a result, fluctuations of an order of magnitude are seen below $E_{\gamma} \approx 5 \mathrm{MeV}$ in Fig. 7(a). Furthermore, deviations from the standard $\gamma \mathrm{SF}$ are also expected due to the presence of quadrupole transitions. It should be noted that ${ }^{64} \mathrm{Ni}$ has many excited states with spin $I=2$ below $E_{x} \approx 4 \mathrm{MeV}$, which can decay to the ground state with quadrupole radiation. Since the $\gamma \mathrm{SF}$ is extracted assuming the emission of dipole radiation

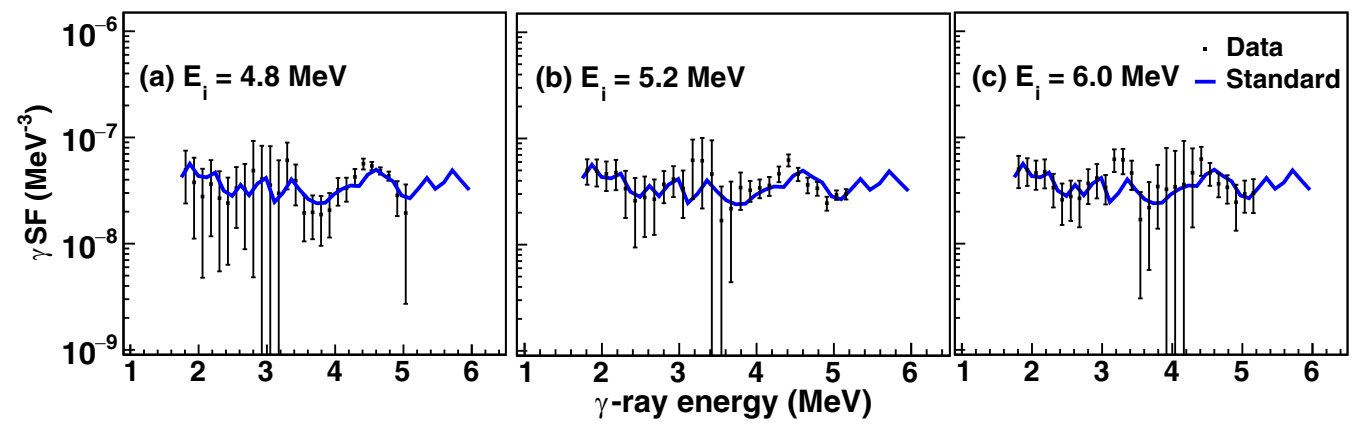

FIG. 6. $\gamma$ strength from several initial excitation energies in ${ }^{64} \mathrm{Ni}$. The results are compared to the standard $\gamma \mathrm{SF}$ (solid line). Each initial excitation energy bin has a width of $248 \mathrm{keV}$. 


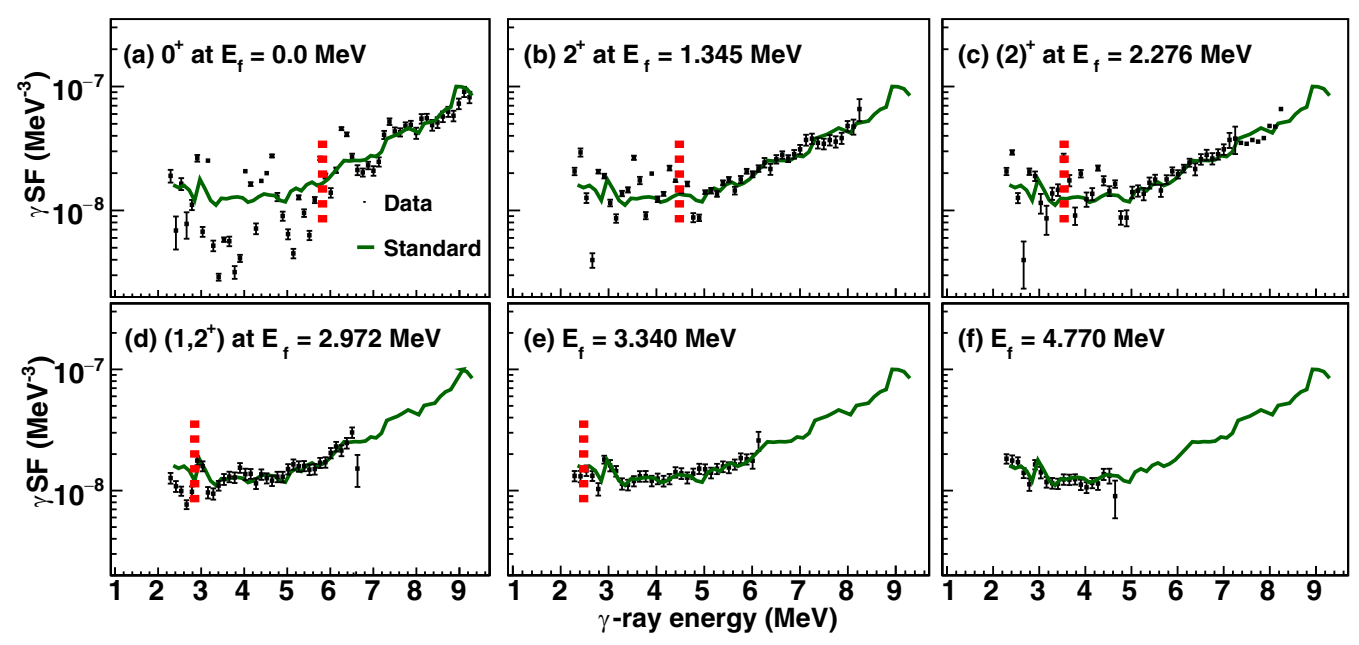

FIG. 7. $\gamma$ strength feeding different final excitation energies for ${ }^{64} \mathrm{Ni}$. The results are compared to the standard $\gamma \mathrm{SF}$ obtained as detailed in Sec. II (solid line). Each final excitation energy bin has a width of $124 \mathrm{keV}$. The vertical dashed line marks the limit $E_{\gamma}=E_{x, \text { min }}-E_{f}$, where $E_{x, \min }$ is the minimum excitation energy used in the extraction of the standard $\gamma \mathrm{SF}$, here $E_{x, \min }=5.82 \mathrm{MeV}$.

in Eq. (2), the estimated strength for a quadrupole transition would be off by a factor of $E_{\gamma}^{2}$.

In the case of ${ }^{65} \mathrm{Ni}$, the results for $f\left(E_{\gamma}, E_{f}\right)$ are in agreement with the standard $\gamma \mathrm{SF}$ for $E_{f}$ at and above $1.92 \mathrm{MeV}$. Above the vertical dashed line, a good agreement is seen for all values of $E_{f}$ considered. Furthermore, even though significant deviations from $f\left(E_{\gamma}\right)$ are seen at $E_{f}=0.69 \mathrm{MeV}$ below the vertical dashed line, the data points are approximately scattered around $f\left(E_{\gamma}\right)$. As seen in Figs. 8(a)-8(c), the resonance-like structure present in the standard $\gamma \mathrm{SF}$ at $E_{\gamma} \approx$ $4.6 \mathrm{MeV}$ is also observed in $f\left(E_{\gamma}, E_{f}\right)$ for the different $E_{f}$ bins considered. The low-energy enhancement is well reproduced, as seen in Figs. 8(e) and 8(f). Below $E_{f}=0.69 \mathrm{MeV}$, larger deviations are observed. Again, a contributing factor to these deviations could be the presence of quadrupole transitions. The results shown in Fig. 8(a) suggest that the ground state is less strongly fed from excitation energies below $\approx 4 \mathrm{MeV}$, where $f\left(E_{\gamma}, E_{f}\right)$ is below $f\left(E_{\gamma}\right)$, while in Fig. 8(b) the strength populating the second excited state at $E_{f}=0.31 \mathrm{MeV}$ is above $f\left(E_{\gamma}\right)$ at $E_{\gamma} \approx 2-3 \mathrm{MeV}$. However, the average strength feeding the ground, first, and second excited states is in agreement with the standard $\gamma \mathrm{SF}$. Note that, as previously shown in Fig. 2, the upbend and resonancelike structure at $E_{\gamma} \approx 4.6 \mathrm{MeV}$ are seen even when the decay to those states is excluded. In conclusion, the overall agreement between the standard $\gamma \mathrm{SF}$ and the various $f\left(E_{\gamma}, E_{f}\right)$ shown in Fig. 8 indicates that no clear dependence on final excitation energy is observed in the $\gamma \mathrm{SF}$ of ${ }^{65} \mathrm{Ni}$ above $E_{f} \approx$ $0.69 \mathrm{MeV}$.

\section{FLUCTUATIONS OF THE $\gamma \mathrm{SF}$}

Validating the gBA hypothesis becomes especially difficult when strong Porter-Thomas fluctuations are present, as is
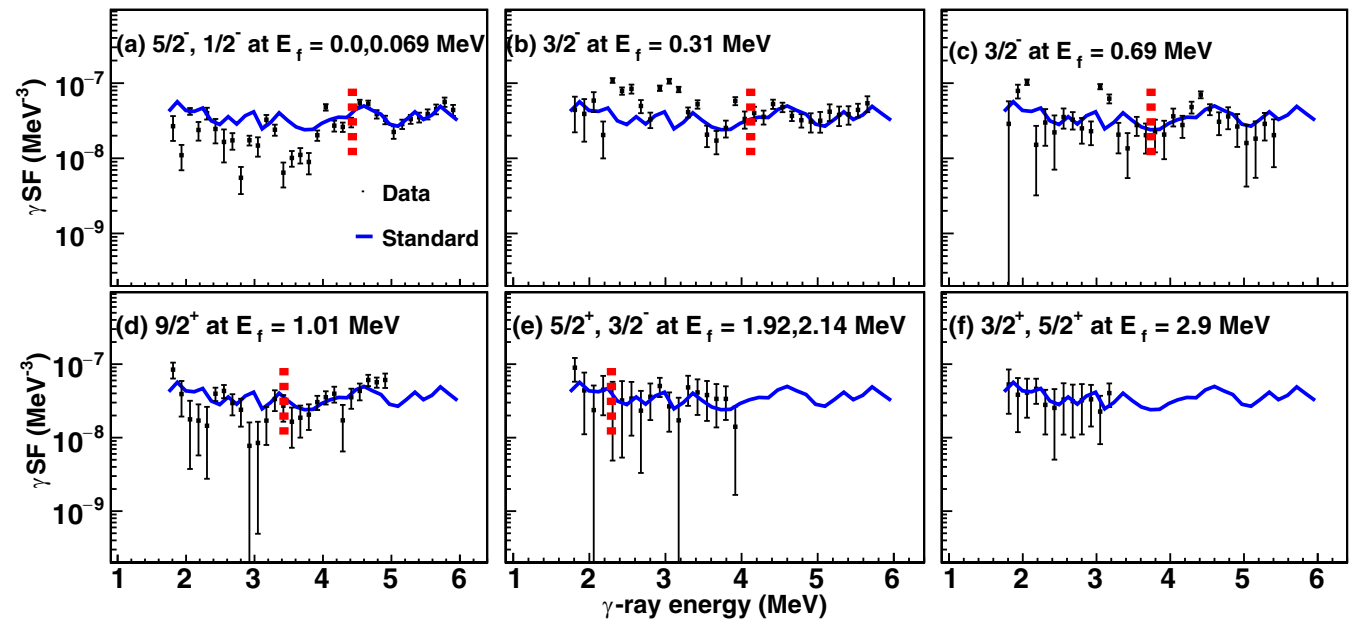

FIG. 8. $\gamma$ strength feeding different final excitation energies for ${ }^{65} \mathrm{Ni}$. The results are compared to the standard $\gamma \mathrm{SF}$ (solid line). Each final excitation energy bin has a width of $248 \mathrm{keV}$. The vertical dashed line marks the limit $E_{\gamma}=E_{x, \min }-E_{f}$, where $E_{x, \text { min }}$ is the minimum excitation energy used in the extraction of the standard $\gamma \mathrm{SF}$, here $E_{x, \min }=4.43 \mathrm{MeV}$. 


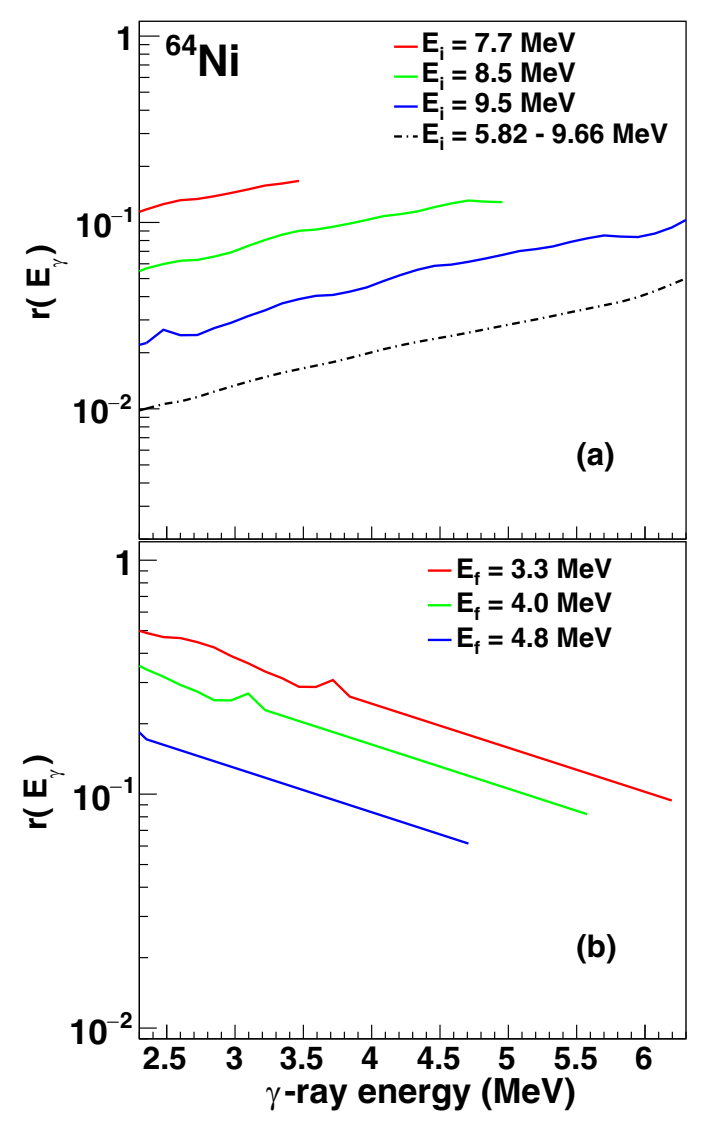

FIG. 9. Fluctuations $r\left(E_{\gamma}\right)$ in ${ }^{64} \mathrm{Ni}$, with $r=\sqrt{2 / n}$ : (a) the results for a specific initial energy bin, obtained with $n=n\left(E_{i}, E_{\gamma}\right)$ as given by Eq. (10), and (b) the equivalent results for a final excitation energy bin. The results are compared to $r\left(E_{\gamma}\right)$ for the standard $\gamma \mathrm{SF}$, obtained for a wider range of initial excitation energies.

often the case for light and medium mass nuclei. In ${ }^{64,65} \mathrm{Ni}$, the neutron separation energies are $S_{n}=9.658 \mathrm{MeV}$ and $S_{n}=6.098 \mathrm{MeV}$, respectively. At these energies, the NLD is $\approx 2600$ levels $/ \mathrm{MeV}$ for ${ }^{64} \mathrm{Ni}$ and $\approx 1100$ levels $/ \mathrm{MeV}$ for ${ }^{65} \mathrm{Ni}$. In contrast, heavier nuclei such as ${ }^{238} \mathrm{~Np}$ present a much larger number of accessible nuclear states, with a NLD of 43 million levels $/ \mathrm{MeV}$ at $S_{n}=5.488 \mathrm{MeV}$. As shown in Ref. [13], Porter-Thomas fluctuations are not significant for ${ }^{238} \mathrm{~Np}$, but they are expected to be larger for lighter nuclei. It is therefore important to estimate more quantitatively if this is the case for ${ }^{64,65} \mathrm{Ni}$ before extracting conclusions regarding the validity of the $\mathrm{gBA}$ in these nuclei.

Following the procedure described in Ref. [13], we here assume that the fluctuations in the $\gamma \mathrm{SF}$ follow the $\chi_{v}^{2}$ distribution, with $v$ equal to the number of transitions, $n$, included in the averaging for a specific $E_{\gamma}$. For a $\chi_{\nu}^{2}$ distribution we define the ratio $r$ between the deviation $\sigma$ and the average $\mu$ as $r=\sigma / \mu=\sqrt{2 / \nu}$. Using the experimental results for the NLD of ${ }^{64,65} \mathrm{Ni}$, we count the number of expected transitions from an initial to a final excitation energy bin $n\left(E_{\gamma}\right)$, and with $v=n\left(E_{\gamma}\right)$ we obtain $r\left(E_{\gamma}\right)=\sqrt{2 / n\left(E_{\gamma}\right)}$. To improve the averaging, a large region of the primary matrix was used as in the Oslo method. The number of transitions was

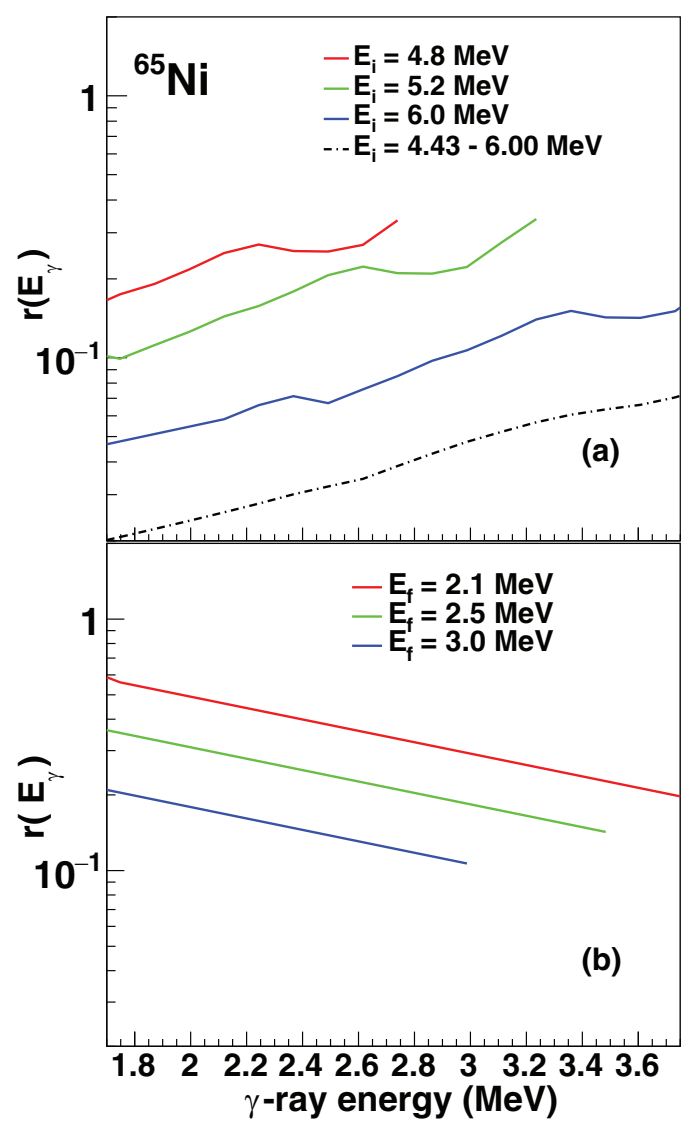

FIG. 10. Fluctuations $r\left(E_{\gamma}\right)$ in ${ }^{65} \mathrm{Ni}$, with $r=\sqrt{2 / n}$. See text in Fig. 9.

estimated as

$$
\begin{aligned}
n\left(E_{\gamma}\right)= & \Delta E^{2} \sum_{E_{i}=E_{i, \min }}^{E_{i, \max }} \sum_{I \pi} \sum_{\delta=-1}^{1} \sum_{\pi^{\prime}} \rho\left(E_{i}, I, \pi\right) \rho \\
& \times\left(E_{i}-E_{\gamma}, I+\delta, \pi^{\prime}\right),
\end{aligned}
$$

where $\Delta E$ is the bin width for the excitation energy, $I$ and $\pi$ are the spin and parity of the initial state, and $\pi^{\prime}$ is the parity of the final state. Note that the sum over $\delta$ runs from -1 to +1 , corresponding to dipole radiation. For a decay from a specific $E_{i}$ bin, the number of transitions is

$$
\begin{aligned}
n\left(E_{\gamma}, E_{i}\right)= & \Delta E^{2} \sum_{I \pi} \sum_{\delta=-1}^{1} \sum_{\pi^{\prime}} \rho\left(E_{i}, I, \pi\right) \rho \\
& \times\left(E_{i}-E_{\gamma}, I+\delta, \pi^{\prime}\right) .
\end{aligned}
$$

In a similar way, the number of transitions feeding a given final excitation energy bin $E_{f}=E_{i}-E_{\gamma}$ was obtained.

Figures 9 and 10 show the expected fluctuations in the $\gamma$ decay of ${ }^{64,65} \mathrm{Ni}$ as a function of $\gamma$-ray energy $r\left(E_{\gamma}\right)$. Figures 9(a) and 10(a) display the fluctuations from a given $E_{i}$ bin in comparison with the results for the standard Oslo method, obtained for $E_{i}=5.82-9.66 \mathrm{MeV}$ for ${ }^{64} \mathrm{Ni}$ and $E_{i}=$ 4.43-6.0 MeV for ${ }^{65} \mathrm{Ni}$, as indicated in Sec. II A. To study the fluctuations within the quasicontinuum, the decays for a given $E_{i}$ here studied correspond to $E_{f}>3 \mathrm{MeV}$. In addition, the analysis of the fluctuations in the feeding of a given $E_{f}$ 


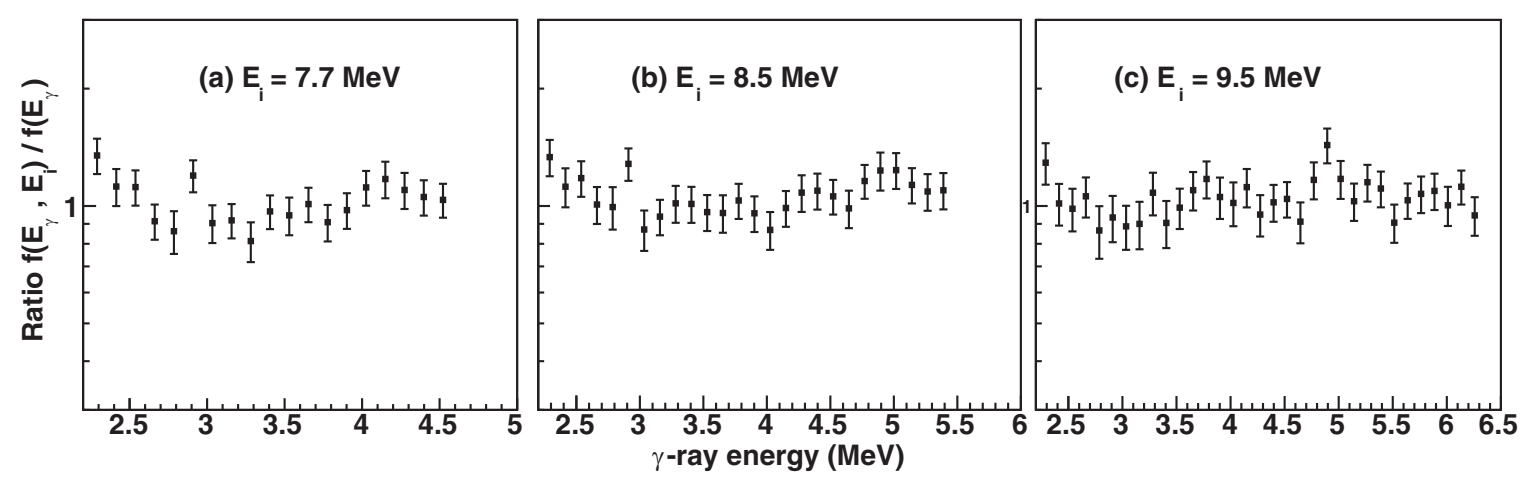

FIG. 11. Ratio of $f\left(E_{\gamma}, E_{i}\right)$ for ${ }^{64} \mathrm{Ni}$ with respect to the excitation-energy-independent $\gamma \mathrm{SF}, f\left(E_{\gamma}\right)$. The results are shown for $E_{i}=7.7$, 8.5, and $9.5 \mathrm{MeV}$.

bin is shown in Figs. 9(b) and 10(b). The bin widths for the excitation energies are 124 and $248 \mathrm{keV}$ for ${ }^{64} \mathrm{Ni}$ and ${ }^{65} \mathrm{Ni}$, respectively.

For a given initial excitation energy, $r\left(E_{\gamma}\right)$ increases exponentially with $E_{\gamma}$ for both ${ }^{64} \mathrm{Ni}$ and ${ }^{65} \mathrm{Ni}$. Note that, for $E_{i}$ close to $S_{n}, r\left(E_{\gamma}\right)$ ranges from $\approx 0.01$ to $\approx 0.10 \mathrm{in}{ }^{64} \mathrm{Ni}$ and from $\approx 0.06$ to $\approx 0.17$ in ${ }^{65} \mathrm{Ni}$. As the initial excitation energy decreases, fewer nuclear states are present and therefore fewer transitions are observed, resulting in larger fluctuations. For example, $r\left(E_{\gamma}\right)$ for ${ }^{64} \mathrm{Ni}$ from $E_{i}=7.7 \mathrm{MeV}$ is about four times higher than from $E_{i}=9.5 \mathrm{MeV}$ for a given $E_{\gamma}$.

The results for the standard $\gamma \mathrm{SF}$ obtained for a wider range of initial excitation energies contain a better averaging over transitions and therefore much smaller fluctuations. For instance, $r\left(E_{\gamma}\right)$ for ${ }^{65} \mathrm{Ni}$ obtained for $E_{i}=4.43-6.00 \mathrm{MeV}$ (the $E_{i}$ range for the standard $\gamma \mathrm{SF}$ ) is about 8 times lower than the $r\left(E_{\gamma}\right)$ obtained when only transitions from the $E_{i}=$ $4.8 \mathrm{MeV}$ bin are considered.

As seen in Figs. 9(b) and 10(b), the fluctuations decrease exponentially with $E_{\gamma}$ and, as expected, they increase as $E_{f}$ decreases, since fewer states are available and therefore fewer transitions take place. For instance, in ${ }^{64} \mathrm{Ni}$ at $E_{f}=3.3 \mathrm{MeV}$, the value of $r\left(E_{\gamma}\right)$ is about three times larger than for decays to $E_{f}=4.8 \mathrm{MeV}$ for a given $E_{\gamma}$.

In addition, the fluctuations in the $\gamma \mathrm{SF}$ were studied by comparing the results for the $\gamma$ strength for a given initial and final excitation energy $f\left(E_{\gamma}, E_{i}\right)$ and $f\left(E_{\gamma}, E_{f}\right)$ with the $E_{x}$-independent $\gamma \mathrm{SF}, f\left(E_{\gamma}\right)$ [15]. In particular, the ratios of $f\left(E_{\gamma}, E_{i}\right)$ and $f\left(E_{\gamma}, E_{f}\right)$ to the average $f\left(E_{\gamma}\right)$ were obtained:

$$
R\left(E_{\gamma}, E_{x}\right)=\frac{f\left(E_{\gamma}, E_{x}\right)}{f\left(E_{\gamma}\right)},
$$

where $x=i, f$ for initial or final excitation energies. The analysis was done for the ${ }^{64} \mathrm{Ni}$ data, with better statistics. First, the fluctuations were studied from $R\left(E_{\gamma}, E_{i}\right)$ with $E_{i}=7.7$, 8.5 , and $9.5 \mathrm{MeV}$, where the level density is $\approx 500,800$, and 1200 levels $/ \mathrm{MeV}$, respectively. To analyze the impact of fluctuations in decays within the quasicontinuum, only decays with $E_{f}=E_{i}+E_{\gamma}>3 \mathrm{MeV}$ were included (with $\rho\left(E_{f}\right)>$ 10 levels/MeV). The results, shown in Fig. 11, are clearly scattered around $R\left(E_{\gamma}, E_{i}\right)=1$, with the data points mostly contained in an $\approx 15 \%$ band around $R\left(E_{\gamma}, E_{i}\right)=1$. When all data points are considered, the average ratio is 1.03. Furthermore, for all $E_{i}$ considered, the deviations from $R\left(E_{\gamma}, E_{i}\right)=$ 1 are less than $7 \%$ on average. In other words, the results are consistent with the excitation-energy-independent $\gamma \mathrm{SF}$ within $\approx 7 \%$ and the deviations can be interpreted as remnants of Porter-Thomas fluctuations, in agreement with the observations for ${ }^{46} \mathrm{Ti}$ in Ref. [15].

To study the impact of decays to low-lying states in the observed fluctuations, the ratio $R\left(E_{\gamma}, E_{f}\right)$ was obtained for $E_{f}=0,1.345$, and $3.340 \mathrm{MeV}$ and the results are shown in Fig. 12. With $E_{f}=0 \mathrm{MeV}$ and $E_{\gamma}=E_{i}>6.4 \mathrm{MeV}$ the ratio
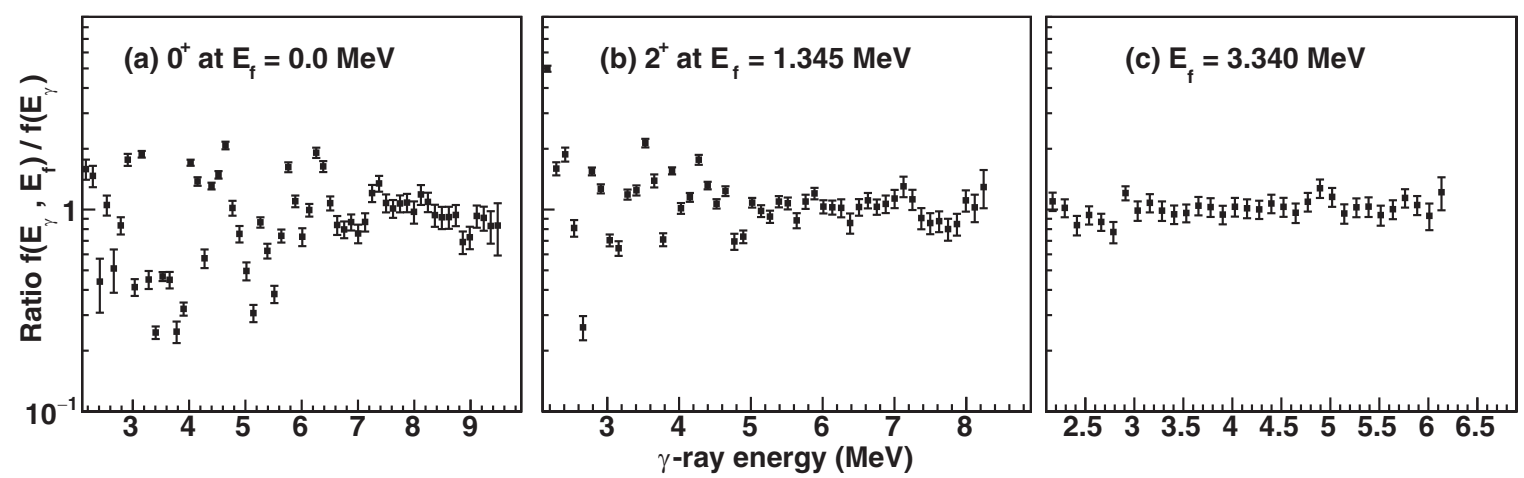

FIG. 12. Ratio of $f\left(E_{\gamma}, E_{f}\right)$ for ${ }^{64} \mathrm{Ni}$ with respect to the excitation-energy-independent $\gamma \mathrm{SF}, f\left(E_{\gamma}\right)$. The results are shown for $E_{f}=0$, 1.35 , and $3.34 \mathrm{MeV}$. 
ranges from 0.71 to 1.28 , with an average of $\approx 0.94$, but its fluctuations increase drastically for $E_{\gamma}=E_{i}<6.4 \mathrm{MeV}$. In other words, even though only one state is available in the final excitation energy bin $E_{f}=0 \mathrm{MeV}$, a ratio close to 1 is obtained when averaging over a sufficiently large number of transitions, i.e., when exploring decays from $E_{i}>6.4 \mathrm{MeV}$ where the level density is larger than $\approx 100$ levels/MeV. Similar results are obtained for $E_{f}=1.345 \mathrm{MeV}$ : in this case, the sudden deviations from a ratio equal to 1 are seen at $E_{\gamma} \approx 5 \mathrm{MeV}$, again corresponding to $E_{i} \approx 6.4 \mathrm{MeV}$. For $E_{f}=3.34 \mathrm{MeV}$, the number of states in the final excitation energy bin is larger ( $>10$ levels $/ \mathrm{MeV}$ ) and a ratio closer to 1 is observed all over the measured $\gamma$-ray energy range. This is in agreement with the results from Ref. [15], which showed that for decays within the quasicontinuum the results were consistent with an excitation-energy-independent $\gamma \mathrm{SF}$ for $E_{f}>3 \mathrm{MeV}$.

\section{SUMMARY}

Particle- $\gamma$ coincidence data from Refs. $[19,20]$ was further analyzed to study the statistical behavior of the $\gamma$ SFs of ${ }^{64} \mathrm{Ni}$ and ${ }^{65} \mathrm{Ni}$. The dependence of the $\gamma \mathrm{SF}$ on both initial and final excitation energies was studied. In other words, the validity of the gBA hypothesis was investigated for the present case. In addition, the role of Porter-Thomas fluctuations as a function of excitation and $\gamma$-ray energies was analyzed.

The results suggest that the $\gamma$ SFs of ${ }^{64,65} \mathrm{Ni}$ are independent of the initial excitation energy and in agreement with the $\gamma \mathrm{SF}$ from Refs. $[19,20]$ at initial excitation energies of 5.82-9.66 and 4.43-6.08 MeV and at $\gamma$-ray energies above 2.0 and $1.6 \mathrm{MeV}$, respectively. The $\gamma \mathrm{SF}$ corresponding to the feeding of the ground and first excited states present large deviations from the $\gamma$ SFs obtained with the standard Oslo method unless decays from sufficiently high initial excitation energies are considered, here above $\approx 5.8-6.4 \mathrm{MeV}$ for ${ }^{64} \mathrm{Ni}$ and above $\approx 4.4 \mathrm{MeV}$ for ${ }^{65} \mathrm{Ni}$. These deviations, scattered around the excitation-energy-independent $\gamma \mathrm{SF}$, can be attributed to large Porter-Thomas fluctuations and probably also to the admixture of quadrupole transitions. With the exception of these deviations, the strength feeding a given final excitation energy bin is in good agreement with the assumption of a common $\gamma \mathrm{SF}$ given by the standard Oslo method for all the excitation and $\gamma$-ray energies considered. Therefore, the results presented in this work support the validity of the generalized Brink-Axel hypothesis in ${ }^{64,65} \mathrm{Ni}$, assumed in Refs. [19,20].

\section{ACKNOWLEDGMENTS}

We would like to thank INFN Laboratory Nazionali di Legnaro for providing the ${ }^{64} \mathrm{Ni}$ target. We are also grateful for the financial support received from the Research Council of Norway (NFR). S.S. and G.M.T. acknowledge funding under NFR project Grants No. 210007 and No. 262952/F20. A.C.L. acknowledges financial support from the ERC-STG2014 under Grant No. 637686. Finally, we would like to thank J. C. Müller, A. Semchenkov, and J. Wikne for providing the beam for our experiments.
[1] A. A. Bohr and B. R. Mottelson, in Nuclear Structure (Benjamin, New York, 1969), Vol. 1, pp. 152-158, 169-171, $183-186$.

[2] G. A. Bartholomew et al., in Advances in Nuclear Physics, edited by M. Baranger and E. Vogt (Plenum, New York, 1973), Vol. 7, p. 229.

[3] D. M. Brink, Doctoral thesis, Oxford University, 1955.

[4] P. Axel, Phys. Rev. 126, 671 (1962).

[5] A. C. Larsen and S. Goriely, Phys. Rev. C 82, 014318 (2010).

[6] I. Daoutidis and S. Goriely, Phys. Rev. C 86, 034328 (2012).

[7] M. Arnould, S. Goriely, and K. Takahashi, Phys. Rep. 450, 97 (2007).

[8] F. Sokolov, K. Fukuda, and H. P. Nawada, Thorium Fuel Cycle Potential Benefits and Challenges, TECDOC 1450 (IAEA, Vienna, 2005).

[9] S. Raman, O. Shahal, and G. G. Slaughter, Phys. Rev. C 23, 2794(R) (1981).

[10] M. Krtička, F. Bečváŕ, J. Honzátko, I. Tomandl, M. Heil, F. Käppeler, R. Reifarth, F. Voss, and K. Wisshak, Phys. Rev. Lett. 92, 172501 (2004).

[11] A. Schiller, A. Voinov, E. Algin, J. A. Becker, L. A. Bernstein, P. E. Garrett, M. Guttormsen, R. O. Nelson, J. Rekstad, and S. Siem, Phys. Lett. B 633, 225 (2006).

[12] M. Guttormsen, A. C. Larsen, A. Görgen, T. Renstrøm, S. Siem, T. G. Tornyi, and G. M. Tveten, Phys. Rev. Lett. 116, 012502 (2016).

[13] M. S. Guttormsen, A. C. Larsen, A. Görgen, T. Renstrøm, S. Siem, T. G. Tornyi, and G. M. Tveten, Is the generalized BrinkAxel hypothesis valid? PoS(INPC2016)062 (2016).
[14] C. E. Porter and R. G. Thomas, Phys. Rev. 104, 483 (1956).

[15] M. Guttormsen, A. C. Larsen, A. Bürger, A. Görgen, S. Harissopulos, M. Kmiecik, T. Konstantinopoulos, M. Krtička, A. Lagoyannis, T. lönnroth, K. Mazurek, M. Norrby, H. T. Nyhus, G. Perdikakis, A. Schiller, S. Siem, A. Spyrou, N. U. H. Syed, H. K. Toft, G. M. Tveten, and A. Voinov, Phys. Rev. C 83, 014312 (2011).

[16] P. E. Koehler, A. C. Larsen, M. Guttormsen, S. Siem, and K. H. Guber, Phys. Rev. C 88, 041305(R) (2013).

[17] A. Spyrou, S. N. Liddick, A. C. Larsen, M. Guttormsen, K. Cooper, A. C. Dombos, D. J. Morrissey, F. Naqvi, G. Perdikakis, S. J. Quinn, T. Renstrøm, J. A. Rodriguez, A. Simon, C. S. Sumithrarachchi, and R. G. T. Zegers, Phys. Rev. Lett. 113, 232502 (2014).

[18] S. N. Liddick, A. Spyrou, B. P. Crider, F. Naqvi, A. C. Larsen, M. Guttormsen, M. Mumpower, R. Surman, G. Perdikakis, D. L. Bleuel, A. Couture, L. Crespo Campo, A. C. Dombos, R. Lewis, S. Mosby, S. Nikas, C. J. Prokop, T. Renstrøm, B. Rubio, S. Siem, and S. J. Quinn, Phys. Rev. Lett. 116, 242502 (2016).

[19] L. Crespo Campo, F. L. Bello Garrote, T. K. Eriksen, A. Görgen, M. Guttormsen, K. Hadynska-Klek, M. Klintefjord, A. C. Larsen, T. Renstrøm, E. Sahin, S. Siem, A. Springer, T. G. Tornyi, and G. M. Tveten, Phys. Rev. C 94, 044321 (2016).

[20] L. C. Campo, A. C. Larsen, F. L. Bello Garrote, T. K. Eriksen, F. Giacoppo, A. Görgen, M. Guttormsen, M. Klintefjord, T. Renstrøm, E. Sahin, S. Siem, T. G. Tornyi, and G. M. Tveten, Phys. Rev. C 96, 014312 (2017).

[21] M. Guttormsen, T. S. Tveter, L. Bergholt, F. Ingebretsen, and J. Rekstad, Nucl. Instrum. Methods Phys. Res. A 374, 371 (1996). 
[22] M. Guttormsen, T. Ramsøy, and J. Rekstad, Nucl. Instrum. Methods Phys. Res. A 255, 518 (1987).

[23] A. Schiller, L. Bergholt, M. Guttormsen, E. Melby, J. Rekstad, and S. Siem, Nucl. Instrum. Methods Phys. Res. A 447, 498 (2000).

[24] A. C. Larsen, M. Guttormsen, M. Krtička, E. Běták, A. Bürger, A. Görgen, H. T. Nyhus, J. Rekstad, A. Schiller, S. Siem, H. K. Toft, G. M. Tveten, A. V. Voinov, and K. Wikan, Phys. Rev. C 83, 034315 (2011).

[25] A. Schiller and M. Thoennessen, At. Data Nucl. Data Tables 93, 549 (2007).
[26] H. A. Bethe, Phys. Rev. C 50, 332 (1936).

[27] J. Kopecky and M. Uhl, Phys. Rev. C 41, 1941 (1990).

[28] T. Belgya et al., Handbook for Calculations of Nuclear Reaction Data, RIPL-2, TECDOC 1506 (IAEA, Vienna, 2006).

[29] A. C. Larsen, N. Blasi, A. Bracco, F. Camera, T. K. Eriksen, A. Görgen, M. Guttormsen, T. W. Hagen, S. Leoni, B. Million, H. T. Nyhus, T. Renstrøm, S. J. Rose, I. E. Ruud, S. Siem, T. Tornyi, G. M. Tveten, A. V. Voinov, and M. Wiedeking, Phys. Rev. Lett. 111, 242504 (2013).

[30] Data extracted using the NNDC On-Line Data Service from the ENSDF database, 2017, http://www.nndc.bnl.gov/ensdf/ 